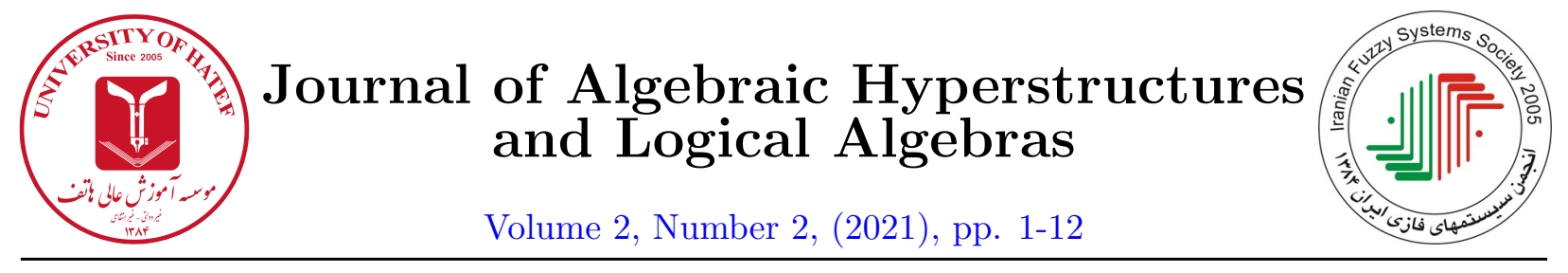

\title{
On nilpotent and solvable pair of polygroups
}

\author{
E. Mohammadzadeh ${ }^{1}$ and F. Mohammadzadeh ${ }^{2}$ \\ ${ }_{1,2}$ Department of Mathematics, Faculty of Science, Payame Noor University, P.O. Box 19395-3697, \\ Tehran, Iran
}

mohamadzadeh36@gmail.com, mohamadzadef464@gmail.com

\begin{abstract}
In this paper, we introduce nilpotent and solvable pair of polygroups. By a pair of polygroups $(P, N)$, we mean a polygroup $P$ with a normal sub-polygroup $N$ of $P$. In addition, we obtain a necessary and sufficient condition between nilpotency (solvability) of pair of polygroups and pair of groups. In particular, we extend the theory of groups to a pair of polygroups. Finally, we study the relationship between nilpotent and solvable pair of polygroups.
\end{abstract}

\section{Article Information}

Corresponding Author:

E. Mohammadzadeh;

Received: April 2021;

Revised: May 2021;

Accepted: June 2021;

Paper type: Original.

\section{Keywords:}

Polygroups, pair of polygroups, solvable pair of polygroups, nilpotent pair of polygroups.

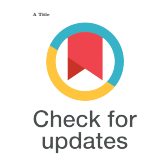

\section{Introduction}

Pair of groups has some applications in group theory, for instance, Ellis $[\underline{\nabla}, \mathbf{g}]$ introduced the notion of Schur multiplier and capability of pair of groups and obtained some results. Also, Hassanzadeh [12] introduced the concept of nilpotency for pair of groups. By a pair of groups $(G, N)$ we mean a group $G$ with a normal subgroup $N$. Clearly, the nilpotency of a group $G$ implies the nilpotency of $(G, N)$. Also, if $(G, N)$ is nilpotent, then so is $N$. A pair of groups is nilpotent if and only if the lower (upper) central series terminates at finite steps.

The theory of algebraic hyperstructures is a well-established branch in algebraic theory. Application of hyperstructures have been studied in many branches of mathematics such as geometry, automata, probabilities, and so on [3, 4]. Fundamental relation is an important concept in hypergroups. It is a way to connect hypergroups and groups. Koskas [15] defined the fundamental

https://doi.org/10.52547/HATEF.JAHLA.2.2.1 
relation $\beta^{*}$ on hypergroups mainly, it has been studied by Corsini [5, 6] and Fereni [U]]. The fundamental relation $\beta^{*}$ is the smallest equivalence relation on a hypergroup $H$ such that the quotient $H / \beta^{*}$ is a group. In 1934, Marty [16] introduced the concept of polygroups as a special hypergroup. Polygroup theory extends some well-known group results and introduce new topics in polygroups. For example, Nilpotent and solvable polygroups are vital notations of group theory

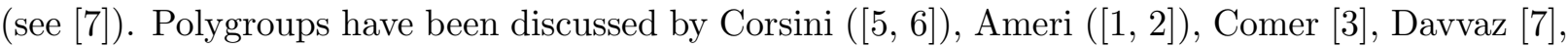
Jafarpour [14], and so on.

Now, in this paper, we study on polygroup analogue of nilpotent pair of groups. In addition, we introduce the notion of solvable pair of groups and ataine some properties of it. Then for a polygroup $P$ and a normal sub-polygroup $N$ we introduce the concept of nilpotent and solvable pair of polygroups. Also, we obtain a necessary and sufficient condition between nilpotent (solvable) pair of polygroups and nilpotent (solvable) pair of groups. A well-known property of nilpotent groups is that the intersection of a normal subgroup of a nilpotent group $G$ by the center of $G$ is non-trivial. Now, we prove the same property on pair of polygroups. Finally, we investigate the relation between nilpotent and solvable pair of polygroups.

\section{Preliminary}

We begin our discussion with some fundamental definitions and results.

A hyperoperation $\circ$ is a mapping from $H \times H$ into the family of non-empty subsets of $H$. A hypergroupoid $(H, \circ)$ is a non-empty set $H$ with a hyperoperation $\circ$. If $A$ and $B$ are non-empty

subsets of $H$, then $A \circ B=\bigcup_{a \in A, b \in B} a \circ b$. Also, we use $x \circ A$ instead of $\{x\} \circ A$ and $A \circ x$ for $A \circ\{x\}$. Generally, the singleton $a$ is identified with its member $a$. The structure $(H, \circ)$ is called a semihypergroup if $a \circ(b \circ c)=(a \circ b) \circ c$ for any $a, b, c \in H$. A semihypergroup $(H, \circ)$ is called a hypergroup if for any $x \in H, x \circ H=H \circ x=H$. The condition that for any $a, b \in H$ there exist $x$ and $y$ such that $a \in b \circ x$ and $a \in y \circ b$, is called the reproduction axiom.

Definition 2.1. [7] An algebraic structure $\left(P, \cdot, e,^{-1}\right)$, where $e \in P$ and ${ }^{-1}$ is an unitary operation on $P$, is called polygroup if for any $x, y, z \in P$ the following conditions hold:

(i) $(x \cdot y) \cdot z=x \cdot(y \cdot z)$,

(ii) $e \cdot x=x \cdot e=x$,

(iii) $x \in y \cdot z \Leftrightarrow y \in x \cdot z^{-1} \Leftrightarrow z \in y^{-1} \cdot x$.

Let $\left(P_{1}, \cdot, e_{1},{ }^{-1}\right)$ and $\left(P_{2}, *, e_{2},{ }^{-1}\right)$ be two polygroups. Then $\left(P_{1} \times P_{2}, \circ\right)$, where $\circ$ is defined as follows, is a polygroup (see [7] $)$.

$$
\left(x_{1}, y_{1}\right) \circ\left(x_{2}, y_{2}\right)=\left\{(x, y) \mid x \in x_{1} \cdot x_{2}, \text { and } y \in y_{1} * y_{2}\right\} .
$$

Note. From now on for a polygroup $\left(P, \cdot, e,^{-1}\right)$ and $x, y \in P$ we use $x y$ instead of $x \cdot y$.

Definition 2.2. [G] A non-empty subset $K$ of a polygroup $P$ is a sub-polygroup of $P$ if for any $a, b \in K$ we have $a b \subseteq K$ and $a^{-1} \in K$. Also, a sub-polygroup $N$ of $P$ is normal if for any $a \in P$, $a^{-1} N a \subseteq N$.

Also, for a sub-polygroup $K$ of $P$ and $x \in P$, denote the left (right) coset of $K$ by $x K(K x)$ and suppose $P / K$ is the set of all left (right) cosets of $K$ in $P$. Note that for a normal subpolygroup $N$ of $P$, we have $N x=x N$ and for all $x, y \in P$ we have $N x y=N z$ for all $z \in x y$. Also, $\left(P / N, \odot, N,^{-1}\right)$ is a polygroup, where

$$
(N x) \odot(N y)=\{N z \mid z \in x y\} \text { and }(N x)^{-1}=N x^{-1}
$$


A polygroup is called commutative if for any $x, y \in P, x y=y x$. Let $(H, \circ)$ and $(H, \star)$ be two hypergroups. A map $f:(H, \circ) \rightarrow(H, \star)$ is called a homomorphism if for any $a, b \in H$, $f(a \circ b) \subseteq f(a) \star f(b)$. Also, $f$ is a good homomorphism if for any $a, b \in H, \quad f(a \circ b)=f(a) \star f(b)$. Let $(H, \circ)$ be a hypergroup. The relation $\rho \subseteq H \times H$ is an equivalence relation and $A, B$ are two non-empty subsets of $H$. Then

$$
A \overline{\bar{\rho}} B \Leftrightarrow a \rho b, \quad \forall a \in A, \forall b \in B .
$$

The relation $\rho$ is called a strongly regular on the left (on the right) if for any $x, y, a \in H$ we have

$$
x \overline{\bar{\rho}} y \Leftrightarrow a \circ x \overline{\bar{\rho}} a \circ y \quad(x \circ a \overline{\bar{\rho}} y \circ a) .
$$

In addition, a strongly regular relation is strongly regular on the right and on the left.

Theorem 2.3. [5] If $(H, \cdot)$ is a hypergroup and $\rho$ is a strongly regular relation on $H$, then $\left(\frac{H}{\rho}, \otimes\right)$ is a group with the following operation:

$$
\rho(x) \otimes \rho(y)=\rho(z), \text { for all } z \in x y .
$$

In [15], Koskas defined the relation $\beta=\bigcup_{n \geq 1} \beta_{n}$, where $\beta_{1}=\{(x, x) ; x \in H\}$ is the diagonal relation on $H$ and

$$
a \beta_{n} b \Leftrightarrow \exists\left(x_{1}, \ldots, x_{n}\right) \in H^{n},\{a, b\} \subseteq \prod_{i=1}^{n} x_{i} .
$$

The transitive closure of $\beta^{*}$, is a strongly regular relation. Freni proved that if $H$ is a hypergroup, then $\beta=\beta^{*}$ (see [10]).

The kernel of the canonical map $\pi: P \longrightarrow \frac{P}{\beta^{*}}$, denote by $\omega_{P}$ or $\omega$, is called the core of $P$. It is easy to prove that $\omega_{P}=\beta^{*}(e)$ and $\beta^{*}(x)^{-1}=\beta^{*}\left(x^{-1}\right)$ for all $x \in P$ (see [7] $)$.

Theorem 2.4. [7] Assume $P_{1}$ and $P_{2}$ are two polygroups. Then $\omega_{P_{1} \times P_{2}}=\omega_{P_{1}} \times \omega_{P_{2}}$.

Theorem 2.5. [[] Let $A$ be a non-empty subset of $P$. Then the intersection of all sub-polygroups of $P$ containing $A$, denoted by $\langle A\rangle$ is equal to $\bigcup\left\{x_{1}^{\epsilon_{1}} \ldots x_{k}^{\epsilon_{k}} \mid x_{i} \in A, k \in \mathbb{N}, \epsilon_{i} \in\{1,-1\}\right\}$.

Definition 2.6. [G] The lower central series of $P$ is the sequence $\cdots \subseteq \gamma_{1}(P) \subseteq \gamma_{0}(P)=P$, where $\gamma_{0}(P)=P$ and for $k>0$,

$$
\gamma_{k+1}(P)=\left\langle\left\{h \in P \mid x y \cap h y x \neq \emptyset \text { such that } x \in \gamma_{k}(P) \text { and } y \in P\right\}\right\rangle .
$$

Also, $P$ is called a nilpotent polygroup if for some $n \in \mathbb{N}, \gamma_{n}(P) \subseteq \omega$. The smallest such $n$ is called a class of $P$.

In [2] it is proved that for any $x, y \in P$ we have

$$
\{h \in P \mid x y \cap h y x \neq \emptyset\}=\{h \in P \mid h \in[x, y]\},
$$

where $[x, y]$ is the commutator of two elements $x, y$ and is defined by $[x, y]=\left\{t \mid t \in x y x^{-1} y^{-1}\right\}$.

Now, we recall the notion of the commutator of subgroups. Let $H$ and $K$ be two subgroups of a group $G$. Then the commutator of $H$ and $K$, denoted by $[H, K]$ is a group generated by $\{[h, k] \mid h \in H, k \in K\}$, where $[h, k]=h k h^{-1} k^{-1}$.

Definition 2.7. [I:] Let $(G, N)$ be a pair of groups. Put $\gamma_{1}(G, N)=N$ and for $i>1 \gamma_{i+1}(G, N)=$ $\left[\gamma_{i}(G, N), G\right]$. Then

$$
N=\gamma_{1}(G, N) \geq \gamma_{2}(G, N) \geq \ldots
$$

is called the lower central series of $(G, N)$. Also, a pair $(G, N)$ is called nilpotent if $\gamma_{n}(G, N)=\{e\}$, where e is the identity element of $G$. 


\section{$3 \quad$ Nilpotent pair of polygroups}

In this section, by the notion of lower central series for a pair of polygroups the concept of nilpotent pair of polygroups is introduced. Also, some basic results are given (see Theorems ??, ?? and ??).

Definition 3.1. Let $N$ be a normal sub-polygroup of $P$. Then $(P, N)$ is called a pair of polygroups. Also, if $H$ and $M$ are sub-polygroups of $P$ and $N$, respectively, then $(H, M)$ is called a sub-pair of $(P, N)$.

Let $N_{1}$ and $N_{2}$ be two sub-polygroups of $P_{1}$ and $P_{2}$, respectively. By a homomorphism from $\left(P_{1}, N_{1}\right)$ to $\left(P_{2}, N_{2}\right)$ we mean a homomorphism from $P_{1}$ to $P_{2}$ which sends $N_{1}$ to $N_{2}$.

Note. From now on, let $(P, N)$ be a pair of polygroups, $k, n \in \mathbb{N}$ and for any $x \in P$ put $\bar{x}=\beta^{*}(x)$.

Definition 3.2. Assume $l_{1}(P, N)=N$ and for any $k>1$,

$$
l_{k}(P, N)=\left\langle\left\{h \in P \mid h \in[x, y] \text { such that } x \in l_{k-1}(P, N) \text { and } y \in P\right\}\right\rangle .
$$

Then $(P, N)$ is called a nilpotent pair of polygroups $(N P)$ if for some $k$ we have $l_{k}(P, N) \subseteq \omega_{N}$. The smallest such $k$ is called the nilpotency class of $(P, N)$.

It is clear that if $P$ is a nilpotent polygroup, then $(P, N)$ is nilpotent.

Example 3.3. (i) Let $P_{1}=\{e, a, b, c\}$. We define $\cdot$ on $P_{1}$ as follows:

\begin{tabular}{|c|c|c|c|c|}
\hline$\cdot$ & $e$ & $a$ & $b$ & $c$ \\
\hline$e$ & $e$ & $a$ & $b$ & $c$ \\
\hline$a$ & $a$ & $a$ & $P_{1}$ & $c$ \\
\hline$b$ & $b$ & $\{e, a, b\}$ & $b$ & $b, c$ \\
\hline$c$ & $c$ & $\{a, c\}$ & $c$ & $P_{1}$ \\
\hline
\end{tabular}

Then $\left(P_{1}, \cdot\right)$ is a polygroup (see [7]). Consider sub-polygroup $N_{1}=\{e, a\}$ of $P_{1}$. It is easy to see that $l_{1}\left(P_{1}, N_{1}\right)=\omega_{N_{1}}=\{e, a\}=N_{1}$ and so a pair $\left(P_{1}, N_{1}\right)$ is an $N P$.

(ii) Let $P_{2}=N_{2}=\{0,1\}$ be the cyclic group of order two. Then $l_{1}\left(P_{2}, N_{2}\right)=N_{2}$ and $l_{2}\left(P_{2}, N_{2}\right)=\omega_{N_{2}}=\{0\}$ and so pair $\left(P_{2}, N_{2}\right)$ is an $N P$.

Theorem 3.4. Let $(P, N)$ be a pair of polygroups and $H$ be a normal sub-polygroup of $P$. If $(P, N)$ is an $N P$, then $(P / H, N / H)$ is an $N P$.

Proof. First, we prove that for any $n \geq 0, l_{n}(P / H, N / H)=l_{n}(P, N) H / H$. We proceed by induction on $n$ and show that

$$
l_{n}\left(\frac{P}{H}, \frac{N}{H}\right) \subseteq \frac{l_{n}(P, N) H}{H} \text { and } l_{n}\left(\frac{(P, N) H}{H}\right) \subseteq l_{n}\left(\frac{P}{H}, \frac{N}{H}\right) .
$$

For $n=1$, the inclusions are obvious. Now, suppose that $l_{n}\left(\frac{P}{H}, \frac{N}{H}\right) \subseteq \frac{l_{n}(P, N) H}{H}$ holds and $y H \in$ $l_{n+1}\left(\frac{P}{H}, \frac{N}{H}\right)$. Therefore, by Definition ??, $y H \in[a H, b H]$, where $a H \in l_{n}\left(\frac{P}{H}, \frac{N}{H}\right)$ and $b H \in \frac{P}{H}$. Then by hypotheses of induction, $a H=a^{\prime} H$ for some $a^{\prime} \in l_{n}(P, N)$. Therefore, $y H \in[a H, b H]=$ $\left[a^{\prime} H, b H\right]$ and so by Definition ??, $y H=y^{\prime} H$ such that $y^{\prime} \in\left[a^{\prime}, b\right] \subseteq l_{n+1}(P, N)$. Hence, $y H \in$ $\frac{l_{n+1}(P, N) H}{H}$.

Conversely, if $y H \in \frac{l_{n+1}(P, N) H}{H}$, then there exists $t \in l_{n+1}(P, N)$ such that $y H=t H$. Therefore, for some $a \in l_{n}(P, N), b \in P$ we have $t \in[a, b]$. Thus, $y H=t H \in[a H, b H]$, where $a H \in l_{n}\left(\frac{P}{H}, \frac{N}{H}\right)$ and so $y H \in l_{n+1}\left(\frac{P}{H}, \frac{N}{H}\right)$. Hence, the proof is complete. 
In what follows we see that the converse of Theorem ?? is not true in general.

Example 3.5. Let $P=S_{3}$ be the symetric group and $N=H=A_{3}$ be the alternative group of degree 3. Then $(P / H, N / H)$ is an $N P$ but $\left(S_{3}, A_{3}\right)$ is not nilpotent because by Definition ??, $\gamma_{1}\left(S_{3}, A_{3}\right)=A_{3}$ and so $\gamma_{2}\left(S_{3}, A_{3}\right)=\left[A_{3}, S_{3}\right] \subseteq A_{3}$. If $\left[A_{3}, S_{3}\right]=\{e\}$, then $S_{3}$ is an abelian group, a contradiction. Thus, $\left[A_{3}, S_{3}\right] \neq\{e\}$. And so $\gamma_{2}\left(S_{3}, A_{3}\right) \neq\{e\}$. By a simillar method for any $n \in \mathbb{N}, \gamma_{n}\left(S_{3}, A_{3}\right) \neq\{e\}$. Hence, $\left(S_{3}, A_{3}\right)$ is not nilpotent.

Corollary 3.6. If $(P, N)$ is an $N P$ and $I \subseteq N$ is a subnormal polygroup of $P$. Then $(P / I, N / I)$ is an NP.

Theorem 3.7. Assume $\left(P_{1}, N_{1}\right)$ and $\left(P_{2}, N_{2}\right)$ are two pairs of polygroups. Then, for all $k \geq 0$ we have:

$$
l_{k}\left(P_{1} \times P_{2}, N_{1} \times N_{2}\right)=l_{k}\left(P_{1}, N_{1}\right) \times l_{k}\left(P_{2}, N_{2}\right) .
$$

Proof. By induction on $k$ we prove that $l_{k}\left(P_{1} \times P_{2}, N_{1} \times N_{2}\right)=l_{k}\left(P_{1}, N_{1}\right) \times l_{k}\left(P_{2}, N_{2}\right)$. The first step of induction holds. Now suppose that $(a, b) \in l_{k}\left(P_{1} \times P_{2}, N_{1} \times N_{2}\right)$, and the result holds for $k-1$. By Definition ??, for some $\left(x_{1}, y_{1}\right) \in l_{k-1}\left(P_{1} \times P_{2}, N_{1} \times N_{2}\right)$ and $\left(z_{1}, z_{2}\right) \in P_{1} \times P_{2}$ we have $(a, b) \in\left[\left(x_{1}, y_{1}\right),\left(z_{1}, z_{2}\right)\right]$. Since $\left[\left(x_{1}, y_{1}\right),\left(z_{1}, z_{2}\right)\right]=\left(\left[x_{1}, z_{1}\right],\left[y_{1}, z_{2}\right]\right)$ we get $a \in\left[x_{1}, z_{1}\right]$ and $b \in\left[y_{1}, z_{2}\right]$. By the hypotheses of induction, we conclude $a \in l_{k-1}\left(P_{1}, N_{1}\right)$ and $b \in l_{k-1}\left(P_{2}, N_{2}\right)$. Hence, $(a, b) \in l_{k}\left(P_{1}, N_{1}\right) \times l_{k}\left(P_{2}, N_{2}\right)$. A similar calculation gives the converse.

Theorem 3.8. Consider $\left(P_{1}, N_{1}\right)$ and $\left(P_{2}, N_{2}\right)$ are two pairs of polygroups. Then $\left(P_{1} \times P_{2}, N_{1} \times N_{2}\right)$ is an $N P$ if and only if $\left(P_{1}, N_{1}\right)$ and $\left(P_{2}, N_{2}\right)$ are $N P$.

Proof. $(\Leftarrow)$ Since $\left(P_{1}, N_{1}\right)$ and $\left(P_{2}, N_{2}\right)$ are NP, then there exist $k_{1}, k_{2} \in \mathbb{N}$ such that $l_{k_{1}}\left(P_{1}, N_{1}\right) \subseteq$ $\omega_{N_{1}}$ and $l_{k_{2}}\left(P_{2}, N_{2}\right) \subseteq \omega_{N_{2}}$. Put $k=\operatorname{lcm}\left\{k_{1}, k_{2}\right\}$. Thus, by Theorems ?? and ??, we get

$$
l_{k}\left(P_{1} \times P_{2}, N_{1} \times N_{2}\right)=l_{k}\left(P_{1}, N_{1}\right) \times l_{k}\left(P_{2}, N_{2}\right) \subseteq l_{k_{1}}\left(P_{1}, N_{1}\right) \times l_{k_{2}}\left(P_{2}, N_{2}\right) \subseteq \omega_{N_{1}} \times \omega_{N_{2}} .
$$

Therefore, $\left(P_{1} \times P_{2}, N_{1} \times N_{2}\right)$ is an NP.

$\Rightarrow$ Since $\left(P_{1} \times P_{2}, N_{1} \times N_{2}\right)$ is an NP, we conclude that there exists $k \in \mathbb{N}$ such that $l_{k}\left(P_{1} \times P_{2}, N_{1} \times\right.$ $\left.N_{2}\right) \subseteq \omega_{N_{1} \times N_{2}}$. Then by Theorems ?? and ??, we have $l_{k}\left(P_{1}, N_{1}\right) \subseteq \omega_{N_{1}}$ and $l_{k}\left(P_{2}, N_{2}\right) \subseteq \omega_{N_{2}}$. Hence, $\left(P_{1}, N_{1}\right)$ and $\left(P_{2}, N_{2}\right)$ are NP.

Example 3.9. Let $\left(P_{1}, N_{1}\right)$ and $\left(P_{2}, N_{2}\right)$ pairs as Example??. Then we define on $P_{1} \times P_{2} \cong P$ as follows:

\begin{tabular}{|c|c|c|c|c|c|c|c|c|}
\hline$\cdot$ & $e$ & $a$ & $b$ & $c$ & $d$ & $f$ & $g$ & $h$ \\
\hline$e$ & $e$ & $a$ & $b$ & $c$ & $d$ & $f$ & $g$ & $h$ \\
\hline$a$ & $a$ & $e$ & $c$ & $b$ & $f$ & $d$ & $h$ & $g$ \\
\hline$b$ & $b$ & $c$ & $b$ & $c$ & $\{e, b, d, g\}$ & $\{a, c, f, h\}$ & $g$ & $h$ \\
\hline$c$ & $c$ & $b$ & $c$ & $b$ & $\{a, c, f, h\}$ & $\{e, b, d, g\}$ & $h$ & $g$ \\
\hline$d$ & $d$ & $f$ & $\{e, b, d\}$ & $a, c, f$ & $d$ & $f$ & $\{d, g\}$ & $\{f, h\}$ \\
\hline$f$ & $f$ & $d$ & $\{a, c, f\}$ & $\{e, b, d\}$ & $f$ & $d$ & $\{f, h\}$ & $\{d, g\}$ \\
\hline$g$ & $g$ & $h$ & $\{b, g\}$ & $\{c, h\}$ & $g$ & $h$ & $\{e, b, d, g\}$ & $\{a, c, f, h\}$ \\
\hline$h$ & $h$ & $g$ & $\{c, h\}$ & $\{b, g\}$ & $h$ & $g$ & $\{a, c, f, h\}$ & $\{e, b, d, g\}$ \\
\hline
\end{tabular}

Now, $(P, \cdot)$ is a polygroup [7]. Also, Theorem ?? implies $\left(P_{1} \times P_{2}, N_{1} \times N_{2}\right)$ is an $N P$. 
Theorem 3.10. Consider $\left(P_{1}, N_{1}\right)$ and $\left(P_{2}, N_{2}\right)$ are two pairs of polygroups and $\phi:\left(P_{1}, N_{1}\right) \longrightarrow$ $\left(P_{2}, N_{2}\right)$ is a good homomorphism. If $\phi$ is one to one and $\left(K_{1}, K_{1} \cap N_{1}\right)$ is a nilpotent sub-polygroup of $\left(P_{1}, N_{1}\right)$, then $\phi\left(K_{1}, K_{1} \cap N_{1}\right)$ is a nilpotent sub-polygroup of $\left(P_{2}, N_{2}\right)$.

Proof. By induction on $n$ we show that $l_{n}\left(\phi\left(K_{1}, K_{1} \cap N_{1}\right)\right)=\phi\left(l_{n}\left(K_{1}, K_{1} \cap N_{1}\right)\right)$. For $n=1$, it is clear. Now, assume $l_{n}\left(\phi\left(K_{1}, K_{1} \cap N_{1}\right)\right)=\phi\left(l_{n}\left(K_{1}, K_{1} \cap N_{1}\right)\right)$ holds and $z \in l_{n+1}\left(\phi\left(K_{1}, K_{1} \cap N_{1}\right)\right)$. Then there exist $x \in l_{n}\left(\phi\left(K_{1}, K_{1} \cap N_{1}\right)\right)$ and $y \in \phi\left(K_{1}\right)$ such that $z \in[x, y]$. By hypotheses of induction $x \in \phi\left(l_{n}\left(K_{1}, K_{1} \cap N_{1}\right)\right)$ and so for some $a \in l_{n}\left(K_{1}, K_{1} \cap N_{1}\right)$ we have $x=\phi(a)$. Also, $y \in \phi\left(K_{1}\right)$ implies that $y=\phi(b)$ for some $b \in K_{1}$. Therefore, $z \in[\phi(a), \phi(b)]=\phi[a, b]$ and so $z \in \phi\left(l_{n+1}\left(K_{1}, K_{1} \cap N_{1}\right)\right)$. Therefore,

$$
l_{n+1}\left(\phi\left(K_{1}, K_{1} \cap N_{1}\right)\right) \subseteq \phi\left(l_{n+1}\left(K_{1}, K_{1} \cap N_{1}\right)\right) .
$$

Conversely, let $z \in \phi\left(l_{n+1}\left(K_{1}, K_{1} \cap N_{1}\right)\right)$. Then for some $c \in l_{n+1}\left(K_{1}, K_{1} \cap N_{1}\right)$ we have $z=\phi(c)$. Since $c \in l_{n+1}\left(K_{1}, K_{1} \cap N_{1}\right)$ we conclude that there exist $a \in l_{n}\left(K_{1}, K_{1} \cap N_{1}\right)$ and $b \in K_{1}$ such that $c \in[a, b]$. Then $z=\phi(c) \in \phi[a, b]=[\phi(a), \phi(b)]$ and so by hypotheses of induction and $a \in l_{n}\left(K_{1}, K_{1} \cap N_{1}\right)$ we have $\phi(a) \in l_{n}\left(\phi\left(K_{1}, K_{1} \cap N_{1}\right)\right)$. Therefore $z \in l_{n+1}\left(\phi\left(K_{1}, K_{1} \cap N_{1}\right)\right)$. Consequently, $l_{n}\left(\phi\left(K_{1}, K_{1} \cap N_{1}\right)\right)=\phi\left(l_{n}\left(K_{1}, K_{1} \cap N_{1}\right)\right)$.

In what follows, consider $\beta_{1}^{*}$ is the restriction of $\beta^{*}$ on $N$. Then by Definitions ??, ?? and Theorem ??, we get a relationship between pair of groups and polygroups.

Theorem 3.11. Let $(G, H)=\left(P / \beta^{*}, N / \beta_{1}^{*}\right)$. Then for any $k \geq 1$ we have:

$$
\gamma_{k}(G, H)=\left\langle\left\{\bar{x} \mid x \in l_{k}(P, N)\right\}\right\rangle(I) .
$$

Proof. We prove by induction on $k$. Since $\gamma_{1}(G, H)=H=\langle\{\bar{x} \mid x \in N\}\rangle$, the case $k=1$ is clear. Now, suppose that $(I)$ holds and $\bar{a} \in\left\langle\left\{\bar{x} \mid x \in l_{k+1}(P, N)\right\}\right\rangle$. Then, $a \in l_{k+1}(P, N)$ and so for some $x \in l_{k}(P, N)$ and $y \in P$ we have $a \in[x, y]$. Hypotheses of induction imply that $\bar{x} \in \gamma_{k}(G, H)$. Then by Theorem ??, $\bar{a}=[\bar{x}, \bar{y}] \in \gamma_{k+1}(G, H)$. Thus, $\left\langle\left\{\beta^{*}(x) \mid x \in l_{k}(P, N)\right\}\right\rangle \subseteq \gamma_{k}(G, H)$.

Conversely, let $\bar{a} \in \gamma_{k+1}(G, H)=\left[\gamma_{k}(G, H), G\right]$. Then $\bar{a}=[\bar{x}, \bar{y}]$ for some $\bar{x} \in \gamma_{k}(G, H), \bar{y} \in G$ and so $\bar{a}=\bar{x} \otimes \bar{y} \otimes \overline{x^{-1}} \otimes \overline{y^{-1}}$ i.e $\bar{x} \otimes \bar{y}=\bar{a} \otimes \bar{y} \otimes \bar{x}$. By hypotheses of induction $x \in l_{k}(P, N)$. Thus, by Theorem ??, there exist $c \in x y$ and $d \in a y x$ such that $\bar{c}=\bar{d}$. Let $t \in y x$. By reproduction axioms of polygroups for $c \in x y$ and $t \in y x$ there exists $u \in P$ such that $c \in u t$. Then $c \in u t \subseteq u y x$. Definition ??(ii), implies that

$$
u \in c(y x)^{-1}=c x^{-1} y^{-1} \subseteq x y x^{-1} y^{-1}=[x, y] .
$$

Then $u \in[x, y]=l_{k+1}(P, N)$. Therefore, by Theorem ??,

$$
\begin{aligned}
\bar{u}=[\bar{x}, \bar{y}] & \Leftrightarrow \bar{u}=\bar{x} \otimes \bar{y} \otimes \overline{x^{-1}} \otimes \overline{y^{-1}} \\
& \Leftrightarrow \bar{u} \otimes \bar{y} \otimes \bar{x}=\bar{x} \otimes \bar{y}=\bar{c}=\bar{d}=\bar{a} \otimes \bar{y} \otimes \bar{x} .
\end{aligned}
$$

Therefore, $\bar{a}=\bar{u} \in\left\langle\left\{\bar{t} \mid t \in l_{k+1}(P, N)\right\}\right\rangle$.

Example 3.12. Consider $P_{1}$ and $N_{1}$ are as Example ??(i). Then $G=P_{1} / \beta^{*}=\{\bar{e}=\bar{a}=\bar{b}=\bar{c}\}$ and $H=N_{1} / \beta_{1}^{*}=\{\bar{e}=\bar{a}\}$. In addition, $\gamma_{1}(G, H)=\{\bar{e}\}$ and $l_{1}\left(P_{1}, N_{1}\right)=\omega_{N_{1}}=\{e, a\}$. Therefore, $\left\langle\left\{\bar{x} \mid x \in l_{1}\left(P_{1}, N_{1}\right)\right\}\right\rangle=\{\bar{e}\}$. Thus, $\gamma_{1}(G, H)=\left\langle\left\{\bar{x} \mid x \in l_{1}(P, N)\right\}\right\rangle$

Now, we are ready to state one of the main results of this section. In fact, we get a connection between NP and nilpotent pair of groups. 
Theorem 3.13. The pair $(P, N)$ is an $N P$ if and only if $\left(\frac{P}{\beta^{*}}, \frac{N}{\beta_{1}^{*}}\right)$ is a nilpotent pair of groups.

Proof. $(\Leftarrow)$ Since $(P, N)$ is an NP, for some $k \in \mathbb{N}$ we have $l_{k}(P, N) \subseteq \omega_{N}$. By Theorem 3.10, we have

$$
\gamma_{k}\left(\frac{P}{\beta^{*}}, \frac{N}{\beta_{1}^{*}}\right)=\left\langle\left\{\bar{t} ; t \in l_{k}(P, N) \subseteq \omega_{N}\right\}\right\rangle=\left\{\bar{t} ; t \in \omega_{N}\right\}=\{\bar{e}\} .
$$

It follows that $\left(\frac{P}{\beta^{*}}, \frac{N}{\beta_{1}^{*}}\right)$ is a nilpotent pair of groups.

$(\Rightarrow)$ Let $\left(\frac{P}{\beta^{*}}, \frac{N}{\beta_{1}^{*}}\right)$ be a nilpotent pair of groups. Then for some $k, \gamma_{k}\left(\frac{P}{\beta^{*}}, \frac{N}{\beta_{1}^{*}}\right)=\{\bar{e}\}$. We show that $l_{k}(P, N) \subseteq \omega_{N}$. Let $t \in l_{k}(P, N)$. By Theorem ??, we get $\bar{t} \in \gamma_{k}\left(\frac{P}{\beta^{*}}, \frac{N}{\beta_{1}^{*}}\right)=\{\bar{e}\}$. Hence, $l_{k}(P, N) \subseteq \omega_{N}$. Therefore, $(P, N)$ is an NP.

Definition 3.14. Assume $Z_{0}(P, N)=\omega_{N}$ and for $i \in \mathbb{N}$,

$$
Z_{i}(P, N)=\left\langle\left\{n \in N \mid n y Z_{i-1}(P, N)=y n Z_{n-1}(P, N) \text { for any } y \in P\right\}\right\rangle .
$$

Then series $Z_{0}(P, N) \subseteq Z_{1}(P, N) \subseteq \ldots$ is called the upper central series of $(P, N)$.

Example 3.15. Let $P=\{e, a, b, c, d, f, g\}$ and $N=\{e, a, b\}$. Define $\cdot$ on $P$ as follows:

\begin{tabular}{|c|c|c|c|c|c|c|c|}
\hline$\cdot$ & $e$ & $a$ & $b$ & $c$ & $d$ & $f$ & $g$ \\
\hline$e$ & $e$ & $a$ & $b$ & $c$ & $d$ & $f$ & $g$ \\
\hline$a$ & $a$ & $e$ & $b$ & $c$ & $d$ & $f$ & $g$ \\
\hline$b$ & $b$ & $b$ & $\{e, a\}$ & $g$ & $f$ & $d$ & $c$ \\
\hline$c$ & $c$ & $c$ & $f$ & $\{e, a\}$ & $g$ & $b$ & $d$ \\
\hline$d$ & $d$ & $d$ & $g$ & $f$ & $\{e, a\}$ & $c$ & $b$ \\
\hline$f$ & $f$ & $f$ & $c$ & $d$ & $b$ & $g$ & $\{e, a\}$ \\
\hline$g$ & $g$ & $g$ & $d$ & $b$ & $c$ & $\{e, a\}$ & $f$ \\
\hline
\end{tabular}

Now, $(P, \cdot)$ is a polygroup [7]. Also, $Z_{0}(P, N)=\omega_{N}=\{e, a\}$ and $Z_{1}(P, N)=\left\langle\left\{n \in N \mid n y Z_{0}(P, N)=\right.\right.$ yn $Z_{0}(P, N)$ for any $\left.\left.y \in P\right\}\right\rangle=\{e, a\}$.

Theorem 3.16. Let for any $x \in P, x x^{-1} \subseteq Z_{i}(P, N)$. Then $Z_{i}(P, N)$ is a normal sub-polygroup of $N$ and $\left[Z_{i+1}(P, N), P\right] \subseteq Z_{i}(P, N)$.

Proof. First, we prove $Z_{i}(P, N)$ is a normal sub-polygroup of $N$. For this let $z \in Z_{i}$ and $n \in$ $N$. Then by Definition ??, we have $z n^{-1} Z_{i-1}(P, N)=n^{-1} z Z_{i-1}(P, N)$. Therefore, by $n n^{-1} \subseteq$ $Z_{i}(P, N)$ we get

$$
n z n^{-1} Z_{i-1}(P, N)=n n^{-1} z Z_{i-1}(P, N) \subseteq n n^{-1} z Z_{i}(P, N)=Z_{i}(P, N) .
$$

So, $n z n^{-1}=n z n^{-1} e \in n z n^{-1} Z_{i-1}(P, N) \subseteq Z_{i}(P, N)$. Hence, $n z n^{-1} \subseteq Z_{i}(P, N)$ and $Z_{i}(P, N)$ is normal.

Now, we show $\left[Z_{i+1}(P, N), P\right] \subseteq Z_{i}(P, N)$. Let $x \in Z_{i+1}(P, N)$ and $y \in P$. Since $Z_{i}(P, N)$ is a normal sub-polygroup, by Definition ??, and $x x^{-1} \subseteq Z_{i}(P, N)$ we conclude that

$$
\begin{aligned}
x^{-1} y^{-1} Z_{i}(P, N)=y^{-1} x^{-1} Z_{i}(P, N) & \Leftrightarrow x y x^{-1} y^{-1} Z_{i}(P, N)=x y y^{-1} x^{-1} Z_{i}(P, N) \\
& \Leftrightarrow[x, y] Z_{i}(P, N)=Z_{i}(P, N) \\
& \Leftrightarrow[x, y] \subseteq Z_{i}(P, N) .
\end{aligned}
$$


Example 3.17. Assume $P$ and $N$ are as Example ??. Then for any $x \in P$ we have $x x^{-1} \subseteq$ $Z_{1}(P, N)$ and $Z_{1}(P, N)=\{e, a\}$ is a normal sub-polygroup of $N$.

Theorem 3.18. Let for any $x \in P$ and $i \in \mathbb{N}, x x^{-1} \subseteq Z_{i}(P, N)$. Then $(P, N)$ is an $N P$ if and only if $Z_{r-1}(P, N)=N$, for some $r>0$.

Proof. $(\Leftarrow)$ It is enough to show that there exists $i \in \mathbb{N}$ such that $l_{i}(P, N) \subseteq \omega_{N}$. We argue by induction on $i$ that for any $i \in \mathbb{N}, l_{i}(P, N) \subseteq Z_{r-i}(P, N)$. For $i=1$, we have $l_{1}(P, N)=$ $N=Z_{r-1}(P, N)=N$. Now, assume $l_{i}(P, N) \subseteq Z_{r-i}(P, N)$ and $a \in l_{i+1}(P, N)$. Then for some $x \in l_{i}(P, N)$ and $y \in P$ we have $a \in[x, y]$. By hypotheses of induction and Theorem ??, $a \in Z_{r-i-1}(P, N)$. Then $l_{i}(P, N) \subseteq Z_{r-i}(P, N)$. If $i=r$, then $l_{r}(P, N) \subseteq Z_{0}(P, N)=\omega_{N}$ and so $(P, N)$ is an NP.

$(\Rightarrow)$ By induction we show that $l_{r-i}(P, N) \subseteq Z_{i}(P, N)$, for any $0 \leq i \leq n$. For $i=0$, we have $l_{r}(P, N) \subseteq Z_{0}(P, N)=\omega_{N}$. Let $l_{r-i}(P, N) \subseteq Z_{i}(P, N)$ and $a \in l_{r-i-1}(P, N)$ and $b \in P$. Then by Definition ??, $[a, b] \subseteq l_{r-i}(P, N)$. The hypothesis of induction implies $[a, b] \subseteq Z_{i}(P, N)$. Therefore, $a b Z_{i}(P, N)=b a Z_{i}(P, N)$ and so by Definition ??, $a \in Z_{i+1}(P, N)$ i.e for every $i \in \mathbb{N}$, $l_{r-i}(P, N) \subseteq Z_{i}(P, N)$. If $i=r-1$, then $Z_{r-1}(P, N)=N$.

Example 3.19. Let $P_{1}$ and $N_{1}$ be as for Example??. Then pair $\left(P_{1}, N_{1}\right)$ is an $N P$ and $Z_{0}\left(P_{1}, N_{1}\right)=$ $\omega_{N_{1}}=N_{1}$.

Now, we generalize a well-known property of nilpotent groups in pair of polygroups.

Theorem 3.20. Assume for any $x \in P, x x^{-1} \subseteq Z_{i}(P, N)$ and $M$ is a non-trivial normal subpolygroup of $P$ such that $M \cap N \neq \omega_{N}$. Then $(P, N)$ is an $N P$ implies that $M \cap Z(P, N) \neq \omega_{N}$.

Proof. By Theorem ??, there exists a positive integer $c$ such that $N=Z_{c}(P, N)$. It follows $M \cap Z_{c}(P, N) \neq \omega_{N}$. Let $i$, be a less integer such that $M \cap Z_{i}(P, N) \neq \omega_{N}$. Now, by Theorem ??, we have

$$
\left[M \cap Z_{i}(P, N), P\right]<M \cap Z_{i-1}(P, N)=\omega_{N} .
$$

By Definition ??, $M \cap Z_{i}(P, N)<M \cap Z_{1}(P, N)$. Hence,

$$
M \cap Z_{i}(P, N)<M \cap Z_{1}(P, N)<M \cap Z_{i}(P, N) .
$$

Therefore, $M \cap Z_{1}(P, N)=M \cap Z_{i}(P, N) \neq \omega_{N}$.

Theorem 3.21. [7] Let $(G,$.$) be a group. Then \left(P_{G}, \circ, e,{ }^{-1}\right)$ is a polygroup, where $P_{G}=G \cup\{a\}$, $a \notin G$ and $\circ$ is defined as follows:

(1) $a \circ a=e$,

(2) $e \circ x=x \circ e=x, \forall x \in G$,

(3) $a \circ x=x \circ a=x, \forall x \in G-\{e, a\}$,

(4) $x \circ y=x . y, \forall(x, y) \in G^{2} ; y \neq x^{-1}$,

(5) $x \circ x^{-1}=x^{-1} \circ x=\{e, a\}, \forall x \in G-\{e, a\}$.

In addition, $P_{G}$ is a nilpotent polygroup if and only if $G$ is a nilpotent group.

Example 3.22. Assume $G$ is the quaternion group $Q_{8}=\{1,-1, i,-i, j,-j, k,-k\}$. Since $G$ is nilpotent by Theorem ??, we conclude that $\left(P_{G}, \circ, e,^{-1}\right)$ is a nilpotent polygroup and so $\left(P_{G}, N\right)$ is nilpotent too. Put $M=\{1,-1, j,-j, a\}$ and $N=\{1,-1, i,-i, a\}$. Clearly, $\omega_{N}=\{1, a\}$, $M \cap N \neq \omega_{N}$ and $x \circ x^{-1}=\{1, a\} \subseteq Z\left(P_{G}, N\right)=\{1,-1, a\}$, for any $x \in P_{G}$. In addition, $-1 \in M \cap Z\left(P_{G}, N\right)$ and $-1 \notin \omega_{N}$ imply that $M \cap Z(P, N) \neq \omega_{N}$.

Corollary 3.23. If $(P, N)$ is an $N P$ and $N \neq \omega_{N}$, then $Z(P, N) \neq \omega_{N}$. 


\section{Solvable pair of polygroups}

The goal of this section is to define the notion of solvable pair of polygroups. Also, we present some results on this topic. Basically, we get a relationship between solvable pair of polygroups and solvable pair of groups. Finally, we prove that every nilpotent pair of polygroups is solvable. First we define a solvable pair of groups. For a pair of groups $(G, N)$, let $(G, N)^{(0)}=N,(G, N)^{(1)}=$ $[N, G]$ and for $k>1,(G, N)^{(k+1)}=\left[(G, N)^{(k)},(G, N)^{(k)}\right]$. The following normal series is called derived series of $(G, N)$ (see [1]2]),

$$
N \geq(G, N)^{(1)} \geq(G, N)^{(2)} \geq \ldots
$$

Definition 4.1. The pair of groups $(G, N)$ is called solvable if for some $k \in \mathbb{N}$ we have $(G, N)^{(k)}=$ $\{e\}$.

Note that for an abelian group $G$ and a normal subgroup $N$ of $G$ the pair $(G, N)$ is solvable. Since $(G, N)^{(1)}=[N, G]=\{e\}$.

Here we define a solvable pair of polygroups. In addition, we study the connection between solvable pair of polygroups and solvable pair of groups.

Definition 4.2. Assume $i_{1}(P, N)=N$ and for $k>1$,

$$
i_{k}(P, N)=\left\langle\left\{h \in P \mid h \in[x, y] \text { such that } x, y \in i_{k-1}(P, N)\right\}\right\rangle .
$$

Then $(P, N)$ is called solvable pair of polygroups $(S P)$ if $i_{k}(P, N) \subseteq \omega_{N}$.

By the same manipulation of the previous section we can obtain the following results, so the proofs are omitted.

Theorem 4.3. Let $(P, N)$ be a pair of polygroups, $H$ be a normal sub-polygroup of $P$ and $H$ be a sub-polygroup of $N$. Then for any $n \geq 0, i_{n}(P / H, N / H)=i_{n}(P, N) H / H$.

Example 4.4. Assume $P$ is as Example ??, $N=\{e, a, b\}$ and $H=\{e, a\}$. Then $P / H=$ $\{e H=a H, b H, c H, d H, f H, g H\}, N H=\{e H=a H, b H\}$. In addition $i_{1}(P, N)=N=\{e, a, b\}$, $i_{2}(P, N)=\{e, a\}$ and $i_{1}(P / H, N / H)=N / H=\{e H=a H, b H\}, i_{2}(P / H, N / H)=\{e H\}$. Therefore, $i_{2}(P / H, N / H)=\{H\}=i_{2}(P, N) H / H$.

Corollary 4.5. If $(P, N)$ is an $S P$ and $H \subseteq N$, then $(P / H, N / H)$ is an $S P$.

Theorem 4.6. Let $\left(P_{1}, N_{1}\right)$, and $\left(P_{2}, N_{2}\right)$ be two pairs of polygroups. Then, for any $k \geq 0$ we have:

$$
i_{k}\left(P_{1} \times P_{2}, N_{1} \times N_{2}\right)=i_{k}\left(P_{1}, N_{1}\right) \times i_{k}\left(P_{2}, N_{2}\right) .
$$

Corollary 4.7. Let $\left(P_{1}, N_{1}\right)$ and $\left(P_{2}, N_{2}\right)$ be two pairs of polygroups. Then $\left(P_{1} \times P_{2}, N_{1} \times N_{2}\right)$ is an $S P$ if and only if $\left(P_{1}, N_{1}\right)$ and $\left(P_{2}, N_{2}\right)$ are $S P$.

Example 4.8. Let $\left(P_{1}, N_{1}\right),\left(P_{2}, N_{2}\right)$ and $(P, N)$ be as Example ??, Clearly, $i_{1}\left(P_{1}, N_{1}\right)=\omega_{N_{1}}$ and $i_{2}\left(P_{2}, N_{2}\right)=\omega_{N_{2}}$. By Corollary ??, $(P, N)$ is an $S P$.

Theorem 4.9. Let $\left(P_{1}, N_{1}\right)$ and $\left(P_{2}, N_{2}\right)$ be two pairs of polygroups and $\phi:\left(P_{1}, N_{1}\right) \rightarrow\left(P_{2}, N_{2}\right)$ be a good homomorphism. If $\phi$ is one to one and $\left(K_{1}, K_{1} \cap N_{1}\right)$ is a solvable sub-polygroup of $\left(P_{1}, N_{1}\right)$, then $\phi\left(K_{1}, K_{1} \cap N_{1}\right)$ is a solvable sub-polygroup of $\left(P_{2}, N_{2}\right)$. 
Theorem 4.10. Consider $(G, H)=\left(P / \beta^{*}, N / \beta^{*}\right)$. Then for any $k \geq 1$ we have:

$$
(G, H)^{(k)}=\left\langle\left\{\bar{x} \mid x \in i_{k}(P, N)\right\}\right\rangle .
$$

Proof. We prove by induction on $k$. For $k=1$, we have $(G, H)^{(1)}=H=\langle\{\bar{x} \mid x \in N\}\rangle$. Now, suppose that $\bar{a} \in\left\langle\left\{\bar{x} \mid x \in i_{k+1}(P, N)\right\}\right\rangle$. Then, $a \in i_{k+1}(P, N)$ and so by Definition ??, there exist $x, y \in i_{k}(P, N)$ such that $a \in[x, y]$. By hypotheses of induction we have $\bar{x}, \bar{y} \in(G, H)^{(k)}$. It follows that $\bar{a}=[\bar{x}, \bar{y}] \in(G, H)^{(k+1)}$. Therefore, $(G, H)^{(k)} \subseteq\left\langle\left\{\bar{x} \mid x \in i_{k}(P, N)\right\}\right\rangle$.

Conversely, let $\bar{a} \in(G, H)^{(k+1)}=\left[(G, H)^{k},(G, H)^{k}\right]$. Therefore, for some $\bar{x}, \bar{y} \in(G, H)^{(k)}$, $\bar{a}=[\bar{x}, \bar{y}]$. Using hypotheses of induction we have $x, y \in i_{k}(P, N)$. Since $\bar{x} \otimes \bar{y}=\bar{a} \otimes \bar{y} \otimes \bar{x}$, by Theorem ??, we conclude that there exist $c \in x y$ and $d \in$ ayx such that $\bar{c}=\bar{d}$. By the same proof of Theorem ??, there exists $u \in P$ such that $u \in[x, y] \subseteq i_{k+1}(P, N)$. Hence,

$$
\bar{u} \otimes \bar{y} \otimes \bar{x}=\bar{x} \otimes \bar{y}=\bar{c}=\bar{d}=\bar{a} \otimes \bar{y} \otimes \bar{x} .
$$

So we have $\bar{a}=\bar{u} \in\left\langle\left\{\bar{t} ; t \in i_{k+1}(P, N)\right\}\right\rangle$. Therefore, $(G, H)^{(k)} \supseteq\left\langle\left\{\beta^{*}(x) \mid x \in i_{k}(P, N)\right\}\right\rangle$.

Theorem 4.11. Pair $(P, N)$ is an $S P$ if and only if $\left(\frac{P}{\beta^{*}}, \frac{N}{\beta_{1}^{*}}\right)$ is a solvable pair of groups.

Proof. $(\Rightarrow)$ Since $(P, N)$ is a solvable pair of groups $i_{k}(P, N) \subseteq \omega_{N}$, by Theorem ??,

$$
\left(\frac{P}{\beta^{*}}, \frac{N}{\beta_{1}^{*}}\right)^{(k)}=\left\langle\left\{\bar{t} ; t \in i_{k}(P, N) \subseteq \omega_{N}\right\}\right\rangle .
$$

So $\left(\frac{P}{\beta^{*}}, \frac{N}{\beta_{1}^{*}}\right)$ is a solvable pair of groups.

$(\Leftarrow)$ Consider $\left(\frac{P}{\beta^{*}}, \frac{N}{\beta_{1}^{*}}\right)$ is solvable. Then there exists $k \operatorname{such}\left(\frac{P}{\beta^{*}}, \frac{N}{\beta_{1}^{*}}\right)^{(k)}=\bar{e}$. Let $t \in i_{k}(P, N)$. By Theorem ??, $\bar{t} \in\left(\frac{P}{\beta^{*}}, \frac{N}{\beta_{1}^{*}}\right)^{(k)}$. Therefore, $i_{k}(P, N) \subseteq \omega_{N}$. This completes the proof.

Now, we study the connection between nilpotent and solvable pair of polygroups.

Theorem 4.12. Every nilpotent pair of polygroups is solvable.

Proof. Let $(P, N)$ be an NP. It is easy to see that for any $k \in \mathbb{N}, i_{k}(P, N) \subseteq l_{k}(P, N)$. Now, since $(P, N)$ is an NP we get that for some $n, l_{n}(P, N) \subseteq \omega_{N}$ and so $i_{n}(P, N) \subseteq \omega_{N}$. Therefore, $(P, N)$ is an SP.

Example 4.13. Assume $P_{1}$ and $N_{1}$ are as in Example ??. Pair $\left(P_{1}, N_{1}\right)$ is an $N P$ and by Theorem ??, we have $\left(P_{1}, N_{1}\right)$ is an SP. In addition, $\left(\frac{P_{1}}{\beta^{*}}, \frac{N_{1}}{\beta_{1}^{*}}\right)=\frac{P_{1}}{\beta^{*}}=\{\bar{e}\}$ and so $\left(\frac{P_{1}}{\beta^{*}}, \frac{N_{1}}{\beta_{1}^{*}}\right)$ is a solvable pair of groups.

A polygroup is called proper if it is not a group.

In what follows we show that the converse of Theorem ??, is not true in general.

Example 4.14. Let $S_{3}$ be the symmetric group and $A_{3}$ be the alternative group of degree 3. Then by definition of derived series of pair $(G, N)$ we have $\left(S_{3}, A_{3}\right)^{(0)}=A_{3}$ and so $\left(S_{3}, A_{3}\right)^{(1)}=\left[A_{3}, A_{3}\right]=$ $\{e\}$. It follows that $\left(S_{3}, A_{3}\right)$ is solvable. But by Example ??, $\left(S_{3}, A_{3}\right)$ is not nilpotent.

Theorem 4.15. Every proper pair of polygroups of order less than 61 is solvable.

Proof. Let $(P, N)$ be a proper pair of polygroups of order less than 61 . Then, $\left(P / \beta^{*}, N / \beta^{*}\right)$ is a pair of groups of order less than 60 that is solvable (see [13]). Therefore, Theorem ??, follows that $(P, N)$ is an $\mathrm{SP}$. 


\section{Conclusion}

In this paper, for a polygroup $P$ and a normal sub-polygroup $N$ of $P$ the notions of nilpotent and solvable pairs of polygroups $(P, N)$ were defined. Moreover, the notion of nilpotent pair of polygroups $(P, N)$ as a generalization of nilpotent polygroup was introduced. Some examples have been used to clarify the concept of nilpotent (solvable) pair of polygroup. In addition, a connection between nilpotent (solvable) pair of groups and nilpotent (solvable) pairs of polygroup was obtained. Especially, the relationship between nilpotent and solvable pair of polygroups were investigated. This work can be used on Engel pair of polygroups, too.

\section{References}

[1] R. Ameri, On categories of hypergroups and hypermodules, Journal of Discrete Mathematical Sciences and Cryptography, 6 (2003), 121-132.

[2] R. Ameri, E. Mohammadzadeh, Engel groups derived from hypergroups, European Journal of Cambinatorics, 44 (2015), 191-197.

[3] S.D. Comer, Extention of polygroups by polygroups and their representations using colour schemes, Lecture Notes in Meth, No 1004, Universal Algebra and Lattice Theory, (1982), 91-103.

[4] S.D. Comer, Hyperstructures assosiated with caracter algebras and colour schemes, in New Ferontiers in Hyperstructures, Hadronic press, palm Harbor, FL, (1995), 49-66.

[5] P. Corsini, V. Leoreanu, Applications of hyperstructure theory, Kluwer Academic Publishers, Dordrecht, 2003.

[6] P. Corsini, Prolegomena of hypergroup theory, Aviani Editore, Tricesimo, 1993.

[7] B. Davvaz, Polygroup theory and related systems, World Scientific, 2013.

[8] G. Ellis, Capability, homology and central series of a pair of groups, Journal of Algebra, 176 (1996), 31-46.

[9] G. Ellis, The schur multiplier of a pair of groups, Applied Categorical Stractures, 6 (1998), $355-371$.

[10] D. Freni, Une note sur le cuur dun hypergroup et sur la cloture transitive $\beta^{*}$ de $\beta$ (A note on the core of a hypergroup and the transitivitive closure $\beta^{*}$ of $\beta$ ), Annali di Matematica Pura ed Applicata, 8 (1991), 153-156 (in French).

[11] D. Freni, A new charactrization of the drived hypergroup via strongly regular equivalences, Communications in Algebra, 30(8) (2002), 3977-3989.

[12] M. Hassanzadeh, A. Pormirzaei, S. Kayvanfar, On the nilpotency of pair of groups, Southeast Asian Bulletin of Mathematics, 37 (2013), 67-77.

[13] T.W. Hungerford, Graduate tex in mathematics, Springer-Verlag New York, Heidelbery Berlin, 1980. 
[14] M. Jafarpour, H. Aghabozorgi, B. Davvaz, On nilpotent and solvable polygroups, Bulletin of the Iranian Mathematical Society, 39(3) (2013), 487-499.

[15] M. Koskas, Groupoides, demi-hypergroups ehypergroupes, Journal de Mathe'matiques Pures et Applique'es, 49 (1970), 155-192.

[16] F. Marty, Sur une Generalization de la Notion de Groupe, in: 8th Congress Mathematics Scandenaves, Stockholm, Sweden, (1934), 45-49. 